\title{
ОБ ИСПОЛЬЗОВАНИИ МАТРИЦЫ СВЯЗИ ПРИ ОПИСАНИИ ПОЛОСНО-ПРОПУСКАЮЩИХ ФИЛЬТРОВ
}

\author{
А. В. ЗАХАРОВ, С. Н. ЛИТВИНЦЕВ, Л. С. ПИНЧУК
}

Национальный технический университет Украиньл «Киевский политехнический институт им. Игоря Сикорского», Украина, Киев, 03056, пр-т Победы 37

\begin{abstract}
Аннотация. В данной статье показано, что возможности матрицы связи $[m]$ при описании микроволновых полосно-пропускающих фильтров (ППФ) ограничены. Существующая процедура вычисления частотных характеристик, основанная на матрице связи, недостоверно описывает физические процессы в реальных ППФ с одним или несколькими резонаторами проходного типа. Причем, эти фильтры могут иметь как простые (магнитные и электрические), так и смешанные коэффициенты связи между резонаторами. Этот факт подтвержден на четырех экспериментальных образцах микрополосковых ППФ. Фильтр третьего порядка с двумя основными и одной перекрестной связью магнитного характера имеет левосторонний нуль передачи, в то время как матрица $[m]$ предписывает правосторонний нуль передачи. Фильтр четвертого порядка с тремя основными и тремя перекрестными связями магнитного характера имеет два разносторонних нуля передачи, в то время как матрица $[m]$ предписывает один правосторонний нуль передачи. Фильтр пятого порядка с четырьмя основными и шестью перекрестными связями магнитного характера имеет АЧХ, близкую к симметричной, если ограничиться уровнем затухания 40 дБ. В то же время, матрица $[m]$ приводит к асимметричной АЧХ этого фильтра с правосторонним нулем передачи. Экспериментальный трехрезонаторный ППФ микрополосковой конструкции со смешанной перекрестной связью обладает симметричной АЧХ с двумя нулями передачи, равноудаленными от центральной частоты полосы пропускания. Использование же матрицы связи приводит к симметричной АЧХ с низкой избирательностью, у которой отсутствуют нули передачи. Дается пояснение имеющим место расхождениям между измеренными характеристиками реальных фильтров и характеристиками, рассчитанными на основе матрицы связи.
\end{abstract}

Ключевые слова: полосно-пропускающий фильтр; матрица связи; перекрестная связь; смешанная связь; нуль передачи; частотная характеристика

\section{1. ВВЕДЕНИЕ}

Микроволновые полосно-пропускающие фильтры (ППФ) играют значительную роль в современных системах беспроводной связи. Они представляют собой набор микроволновых резонаторов, связанных между собой и с нагрузками электромагнитными связями. Существенным шагом в развитии ППФ было введение в их теорию матрицы связи $[m]$, позволяющей по заданным ее элементам определить частотные характеристики фильтра $[1,2]$.
Одно из основных достоинств матрицы $[m]$ заключается в возможности вариации ее элементов с целью получения необходимых частотных характеристик. Подобный анализ осуществляется с применением вычислительных средств и выполняется достаточно быстро. Если структура связи фильтра, включающая расположение резонаторов с указанием связей между ними, определена, то можно осуществить целенаправленный перебор параметров матрицы $[m]$, чтобы добиться максималь- 


\section{БИБЛИОГРАФИЧЕСКИЙ СПИСОК}

1. Atia, A. E.; Williams, A. E. "Narrow-bandpass waveguide filters," IEEE Trans. Microw. Theory Tech., Vol. 20, No. 4, p. 258-265, Apr. 1972. DOI: $\underline{10.1109 /}$ TMTT.1972.1127732.

2. Atia, A.; Williams, A.; Newcomb, R. "Narrow-band multiple-coupled cavity synthesis," IEEE Trans. Circuits Syst., Vol. 21, No. 5, p. 649-655, Sep. 1974. DOI: $10.1109 /$ TCS.1974.1083913.

3. Atia, W. A.; Zaki, K. A.; Atia, A. E. "Synthesis of general topology multiple coupled resonator filters by optimization," IEEE MTT-S Int. Microw. Symp. Dig., 7-12 Jun. 1998, Baltimore, USA. IEEE, 1998, Vol. 2, p. 821-824. DOI: 10.1109/MWSYM.1998.705116.

4. Amari S. "Synthesis of cross-coupled resonator filters using an analytical gradient-based optimization technique," IEEE Trans. Microw. Theory Tech., Vol. 48, No. 9, p. 1559-1564, Sep. 2000. DOI: $10.1109 / 22.869008$.

5. Ланнэ, А.А. Оптимальный синтез линейных электронных схем. М.: Связь, 1978.

6. Levy, R. "Synthesis of general asymmetric singlyand doubly-terminated cross-coupled filters," IEEE Trans. Microw. Theory Tech., Vol. 42, No. 12, p. 2468-2471, Dec. 1994. DOI: $10.1109 / 22.339783$.

7. Levy, R. "Direct synthesis of cascaded quadruplet (CQ) filters," IEEE Trans. Microw. Theory Tech., Vol. 43, No. 12, p. 2940-2945, Dec. 1995. DOI: $\underline{10.1109 / 22.47}$ $\underline{6634}$.

8. Amari, S. "On the maximum number of finite transmission zeros of coupled resonator filters with a given topology," IEEE Microw. Guided Wave Lett., Vol. 9, No. 9, p. 354-356, Sep. 1999. DOI: 10.1109/75.790472.

9. Cameron, R. J. "General coupling matrix synthesis methods for Chebyshev filtering functions," IEEE Trans. Microw. Theory Tech., Vol. 47, No. 4, p. 433-442, Apr. 1999. DOI: $10.1109 / 22.754877$.

10. Cameron, R. J. "Advanced coupling matrix synthesis techniques for microwave filters," IEEE Trans. Microw. Theory Tech., Vol. 51, No. 1, p. 1-10, Jan. 2003. DOI: 10.1109/TMTT.2002.806937. 
11. Hong, J.-S. Microstrip Filters for RF/Microwave Applications, 2nd ed. New York: Wiley, 2011. DOI: 10.1 002/9780470937297.

12. Levy, R.; Petre, P. "Design of CT and CQ filters using approximation and optimization," IEEE Trans. Microw. Theory Tech., Vol. 49, No. 12, p. 2350-2356, Dec. 2001. DOI: 10.1109/22.971620.

13. Ma, K.; Ma, J.-G.; Yeo, K. S.; Do, M. A. “A compact size coupling controllable filter with separate electric and magnetic coupling paths," IEEE Trans. Microw. Theory Tech., Vol. 54, No. 3, p. 1113-1119. 2006. DOI: 10.1109/TMTT.2005.864118.

14. Hoft, M.; Shimamura, T. "Design of symmetric trisection filters for compact low-temperature co-fired ceramic realization," IEEE Trans. Microw. Theory Tech., Vol. 58, No. 1, p. 165-175, Jan. 2010. DOI: $\underline{10.1109 /}$ TMTT.2009.2035870.

15. Zakharov, A.; Rozenko, S.; Ilchenko, M. "Two types of trisection bandpass filters with mixed cross-coupling," IEEE Microw. Wirel. Compon. Lett., Vol. 28, No. 7, p. 585-587, Jul. 2018. DOI: 10.1109/ LMWC.2018.2837905.

16. Zakharov, A.; Ilchenko, M. "Trisection microstrip delay line filter with mixed cross-coupling," IEEE Microw. Wirel. Compon. Lett., Vol. 27, No. 12, p. 1083-1085, Dec. 2017. DOI: 10.1109/LMWC.2017.275 9724.

17. Szydlowski, L.; Lamecki, A.; Mrozowski, M. "Coupled-resonator filters with frequency-dependent couplings: Coupling matrix synthesis," IEEE Microw. Wireless Compon. Lett., Vol. 22, No. 6, p. 312-314, Jun. 2012. DOI: 10.1109/LMWC.2012.2197386.

18. Szydlowski, L.; Leszczynska, N.; Lamecki, A.; Mrozowski, M. "A substrate integrated waveguide (SIW) bandpass filter in a box configuration with frequency-dependent coupling," IEEE Microw. Wireless Compon. Lett., Vol. 22, No. 11, p. 556-558, Nov. 2012. DOI: 10.1109/LMWC.2012.2221690.

19. Szydlowski, L.; Lamecki, A.; Mrozowski, M. "Coupled-resonator waveguide filter in quadruplet topology with frequency-dependent coupling - A design based on coupling matrix," IEEE Microw. Wireless Compon. Lett., Vol. 22, No. 11, p. 553-555, Nov. 2012. DOI: 10.1109/LMWC.2012.2225604.

20. Szydlowski, L.; Leszczynska, N.; Mrozowski, M. "Generalized Chebyshev bandpass filters with frequency-dependent couplings based on stubs," IEEE Trans. Microw. Theory Tech., Vol. 61, No. 10, p. 3601-3612, Oct. 2013. DOI: 10.1109/TMTT.2013.2279 $\underline{777}$.

21. Szydlowski, L.; Jedrzejewski, A.; Mrozowski, M. "A trisection filter design with negative slope of frequency-dependent crosscoupling implemented in substrate integrated waveguide (SIW)," IEEE Microw.
Wireless Compon. Lett., Vol. 23, No. 9, p. 456-458, Sep. 2013. DOI: 10.1109/LMWC.2013.2272611.

22. Szydlowski, L.; Mrozowski, M. "A self-equalized waveguide filter with frequency-dependent (resonant) couplings," IEEE Microw. Wireless Compon. Lett., Vol. 24, No. 11, p. 769-771, Nov. 2014. DOI: 10.1109/LMWC.2014.2303171.

23. Matthaei, G. L.; Jones, E. M. T.; Young, L. Microwave Filters, Impedance-Matching Network, and Coupling Structures. Norwood, MA: Artech House, 1980.

24. Kurzrok, R. M. "General three-resonator filters in waveguide," IEEE Trans. Microw. Theory Tech., Vol. 14, No. 1, p. 46-47, Jan. 1966. DOI: 10.1109/TMTT.1966.112 $\underline{6154}$.

25. Захаров, А.В.; Розенко, С.А. “Дуплексер на основе микрополосковых фильтров, использующих подложки с высокой диэлектрической проницаемостью," Радиотехника и электроника, Т. 57, № 6, с. 713-720, 2012. URI: https://elibrary.ru/item.asp?id=1772 $\underline{6257}$.

26. Kurzrok, R. M. "General four-resonator filters at microwave frequencies," IEEE Trans. Microw. Theory Tech., Vol. 14, No. 6, p. 295-296. 1966. DOI: $10.1109 /$ TMTT.1966.1126254.

27. Захаров, А. В.; Ильченко, М.Е. "Полосно-пропускающие фильтры решетчатого типа на основе полуволновых резонаторов из отрезков симметричных полосковых линий передачи," Радиотехника и электроника, Т. 60, № 7, с. 759-765. 2015. DOI: $10.7868 /$ S0033849415060182.

28. Захаров, А.В.; Ильченко, М.Е.; Пинчук, Л.С. "Коэффициенты связи между ступенчато-импедансными резонаторами в полосковых полосно-пропускающих фильтрах решетчатого типа," Известия вузов. Радиоэлектроника. Т. 57, № 5, C. 35-43, 2014. DOI: 10.20535/S0021347014050045.

29. Захаров, А.В.; Ильченко, М.Е.; Пинчук Л.С. “Зависимость коэффициента связи между четвертьволновыми резонаторами от параметров гребенчатых полосковых фильтров," Известия вузов. Радиоэлектроника, Т. 58, № 6, С. 52-60, 2015. DOI: 10.2053 5/S0021347015060060.

30. Richards, P. I. "Resistor-transmission-line circuits," Proc. IRE, Vol. 36, No. 2, p. 217-220, Feb. 1948. DOI: $10.1109 /$ JRPROC.1948.233274.

31. Riblet, H. J. "General synthesis of quarter-wave impedance transformers," IRE Trans. Microwave Theory Tech., Vol. 5, No. 1, p. 36-43, Jan. 1957. DOI: 10.1109/TMTT.1957.1125088.

32. Matsumoto, A. Microwave Filters and Circuits. Academic Press, 1970. URI: https://www.elsevier.com/ books/microwave-filters-and-circuits/matsumoto/978-0-1 2-027961-6. 